\title{
Kararlı Çoklu Bayes Hipotez Testi Üzerine Bir Not
}

\author{
Uğur Yıldırım ${ }^{1 *}$, Hüseyin Afşer ${ }^{2}$ \\ 1* Adana Alparslan Türkeş Bilim ve Teknoloji Üniversitesi, Mühendislik Fakültesi, Elektrik-Elektonik Mühendislik Bölümü, Adana, Türkiye, (ORCID: 0000-0003- \\ 1131-8893), uyildirim@atu.edu.tr \\ ${ }^{2}$ Adana Alparslan Türkeş Bilim ve Teknoloji Üniversitesi, Mühendislik Fakültesi, Elektrik-Elektonik Mühendislik Bölümü, Adana, Türkiye (ORCID: 0000-0002-6302- \\ 4558), afser@atu.edu.tr
}

(2nd International Conference on Access to Recent Advances in Engineering and Digitalization (ARACONF)-10-12 March 2021)

(DOI: $10.31590 /$ ejosat.899254)

ATIF/REFERENCE: Yıldırım U. \& Afser H. (2021). Kararlı Çoklu Bayes Hipotez Testi Üzerine Bir Not. Avrupa Bilim ve Teknoloji Dergisi, (24), 143-148.

\section{Öz}

Çoklu Bayes hipotez testinde olasılık dağılımlarının bilindiği durumlar için optimum yöntemler bulunmasına karşın gerçek hayata uygulanması zordur. Bu yüzden gerçek dünyada iletişim sırasında kolaylıkla oluşabilecek gürültü vb. kaynaklı veri bozulmarına karşı esneklik kazandıran kararlı algoritmalar kullanılmak zorunludur. Ayrıca kararlılık özelliği, optimal testlerin uygulanabilmesi için kullanılacak kesin bilinmek zorunda olan olasılık dağılımlarına olan bağımlılığı azaltıp, bu olasılık değerlerinin tahmin edilmiş değerlerinin kullanılabilmesine olanak sağlamaktadır. Bu çalışmada kararlı Bayes çoklu hipotez test problem analizleri karşılaştırılmıştır. Bununla birlikte, olasılık dağılımları birbirinden bağımsız ve eşit dağılıma sahip gözlemlerle incelendi. Çoklu Bayes hipotez testi için en yaygın olarak bilinen kararlı yöntem DGL (Devroye, Gyorfi ve Lugasi) metodur. Bu yöntemde gerçek olasılık dağılımları bilinmediği fakat nominal dağılımlar ve gerçek dağılımlar arasındaki uzunluğun $\epsilon$ değerinden küçük olduğunun bilindiği varsayılmıştır. Bu tanım üstel bir hata olasılığını da doğurmuştur ve buradaki hata olasılığının hipotezler arasındaki minimum ikili Chernoff bilgisinden daha düşük olamayacağı da bilinmektedir. DGL yöntemi, tipler metoduna dayalı Afşer tarafından sunulmuş bir analiz ile süreksiz durum için karşılaştırıldı.

Anahtar Kelimeler: Bayes hipotez testi, Tipler metodu, Kararlı hipotez testi, Chernoff bilgisi, Çoklu hipotez testi.

\section{A Note On Robust Multiple Bayesian Hypothesis Testing}

\begin{abstract}
There are exist optimum methods for multiple Bayesian hyothesis testing with known probability distributions but applying this methods to real world is troublesome. Because this tests could gives inaccurate results under small distortions which can easily occur in real world. Also property of robustness gives usage of estimated distributions instead of exact real distributions. In this paper, Bayesian multiple hypothesis problem with independent and identically distributed observations are considered. Robust algorithms are significantly important for solving this kind of problems. The most recognised robust analysis is DGL (Devroye, Gyorfi ve Lugasi) method for multiple Bayesian hypothesis testing. DGL method analyze the case where true distributions of the hypothesis are not known, but the distance between true distributions and nominal distributions are bounded with $\epsilon$. As a consequence of the problem, the achievable error exponent is the minimum pairwise Chernoff information between isolated hypothesis distributions in this setup. In discrete case, DGL method is compered with fresh presented method by Afşer.
\end{abstract}

Keywords: Bayesian hypothesis testing, Method of types, Robust hypothesis testing, Chernoff information, Multiple hypothesis testing.

\footnotetext{
*Sorumlu Yazar: uyildirim@atu.edu.tr
} 


\section{Giriş}

Çoklu hipotez test problemi uzunluğu $n$ olan $X^{n}$ vektörü gözlemlenerek, bu vektörün dağılımını açıklayan $M$ hipotezden, $\mathcal{H}_{1}, \mathcal{H}_{2}, \ldots \mathcal{H}_{\mathcal{M}}$, birinin seçilmesidir. $\mathrm{Bu}$ problemde $X^{n}$ vektörünün elemanları $\quad X=1,2, \ldots, \mathrm{M}$ alfabesindendir, birbirlerinden bağımsızdır ve aynı dağılıma sahiptir. Bu olasılık dağılımları $\mathcal{H}_{1}, \mathcal{H}_{2}, \ldots \mathcal{H}_{\mathcal{M}}$ hipotezleri altında $P_{1}, P_{2}, \ldots, P_{M}$ 'dir [1]. Bu problemde amaç verilen $X^{n}=x^{n}$ gözleminden olasilık dağıllımı en benzer olan hipoteze $\mathcal{H}_{i}, i=1,2, \ldots, M$ karar veren bir test geliştirmektir. Bu makalede olasılık dağılımları $\mathcal{X}$ alfabesine göre tanımlanmıştır, sayılabilir sonludur ve ayrıktır. Testlerin uygulanabilmesi için olasılık uzayı, $M$ ayrışık ve birlikte kapsayıcı bölümlere $\Omega_{1}, \Omega, \ldots, \Omega_{M}$ şeklinde bölünmüştür. Bu bölümlerde eğer $\vec{x} \in \Omega_{i}$ ise $\mathcal{H}_{i}$ hipotezi seçilir. Bu karar mekanizması beraberinde hata olasılı̆gınıda oluşturur. Bu hata olasılığı, $P\left(e \mid \mathcal{H}_{i}\right), \mathcal{H}_{i}$ doğru kabul edildiği ama testin diğer hipotezlerden birisine karar verdiği durum şeklinde tanımlanabilir. Bayes düzeneğinde, $M$ hipotez için hata olasılıkları $P\left(\mathcal{H}_{1}\right), P\left(\mathcal{H}_{2}\right), \ldots, P\left(\mathcal{H}_{\mathcal{M}}\right)$ toplamı aşağıdaki denkleme eşittir.

$$
P(e)=\sum_{i=1}^{M} P\left(e \mid \mathcal{H}_{i}\right) P\left(\mathcal{H}_{i}\right)
$$

Yukarıdaki olasılıklara göre minimizasyon yapan kural sonuçların maksimum sonsal karar kuralıdır. $n$ yeteri kadar büyük olduğunda, önsel efektler ortadan kayboluyor ve maksimum sonsal karar kuralı en yakın komşu kuralına aşağıdaki şekilde dönüşmektedir.

$$
"\left\{\mathcal{H}_{i} \text { hipotezini seç\}, } i=\operatorname{argmin}_{j \in\{1,2, \ldots, M\}} D\left(P_{\vec{x}} \mid P_{j}\right) .\right.
$$

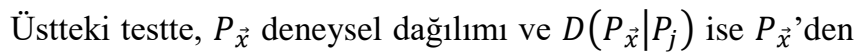
$P_{j}$ 'ye olan Kullback-Leibler(KL) uzaklığını ifade etmektedir. Olasılık uzayı, $M$ ayrık bölüme ağırlık merkezleri $P_{j}, j=$ $1,2, \ldots, M$ olacak şekilde ayrılmıştır. Karar kuralı $P_{\vec{x}}$ ile $P_{j}$ arasındaki KL uzaklığını ölçer ve $\vec{x}$ 'nün en yakın olduğu bölgeye seçilir.

$\mathrm{Bu}$ probleme sunulan en yakın komşu kuralı teorik olarak optimal olmasina rağmen gerçek dünyadaki gürültü, verinin kaybolması gibi dağılımları olumsuz etkileyecek durumlara karşı oldukça duyarlıdır. $\mathrm{Bu}$ duyarlılık gerçek hayata uygulanmasını oldukça zorlaştırmaktadır. Duyarlılığa karşı sunulan çözümler, olasılık dağılımının bilinmesine duyulan gereğin azaltılmasını sağlayan algoritmalar ile mümkün kılınmıştır. $\mathrm{Bu}$ makalede olasılık dağılımlarının bilinmediği fakat bu olasılık dağılımları ile arasındaki uzaklığın $\epsilon$ değerinden küçük olduğunun bilindiği durum için sunulan iki metodun karşılaştırılması ikinci bölümde incelenmiştir.

En yakın komşu kuralının kararlı olmadığı dağılımlarda önemsiz küçük değiişimlerin sonuçlarında felakete sürüklediği Devroye'nin [2] gösterdiği örnekte basit bir şekilde görülebilir. $M=2$ iken özel bir durum olan, Huber'in metodunun sonuçları [3] en yakın komşu kuralında ufak bir değiş̧iklik ve optimal minimax sezinlemesiyle en kötü durumdaki hata olasılıklarını minimize eden bir model aşağıdaki şekilde sunulmuştur.

$$
\prod_{l=1}^{n} \max \left[c, \min \left\{\left(c^{\prime}, \frac{f^{(1)}\left(X_{l}\right)}{f^{(2)}\left(X_{l}\right)}\right\}\right)\right]
$$

Yukarıdaki testte $c$ değeri sabit olup, $c^{\prime}$ değeri nominal yoğunluğa göre değişmektedir. Huber'in testinin dezavantajı verilen sabit değerlerin tam olarak belirlenmek zorunda olması ve bu sabit değerleri belirlemenin problem olabilmesi, özellikle $d>1$ için. Ayrıca, Huber'in sonuçları alfabenin ikiden büyük olduğu durumları kapsamamaktadır ve asimptotik olmayan hata olasılıklarını desteklememektedir.

Bayes kararlı hipotez testine sunulan bir başka yöntem ise Levy [4], [5] tarafindan simetrik dağılımlar için sunulmuştur. Levy'nin kararlı testi yanlış alarm ve hatalı tespit hatalarını, tek bir örnek üzerinden hata olasıllığını minimize etmiştir. Daha sonra ise Gül bu çalışmaları ikili hipotez testi için geliştirmiştir [6]. $\mathrm{Bu}$ yöntemlerin dezavantajları ise çoklu hipotez testine uygulanamaması ve dağılımların simetrik olma zorunluluğudur.

Klasik $P\left(e \mid \mathcal{H}_{i}\right)$ ile $P(e)$ hata analizleri Sanov'un teoremine ve büyük sayıların sapma teorisine dayanmaktadır [7]-[9]. Bu analiz hangi dağılımın hata olasılığının, $P(e)$, üstsel azalma hızının minimum Chernoff uzaklık çiftine eşit olacağını ve hangi hipotez dağılımından geldiğini belirlemeye dayanmaktadır. $\mathrm{Bu}$ üstsel azalma hızının Chernoff uzunluğundan daha fazla olamayacağı bilinmektedir [7], [10]. Chernoff uzunluğu

$$
C(P, Q)=-\min _{\lambda \in[0,1]} \log \left(\sum_{x \in X} Q^{\lambda}(x) Q^{1-\lambda}(x)\right) .
$$

şeklinde tanımlanmıştır.

Bu makalede DGL metodu ve tipler metoduna dayalı analiz ön hazırlık bölümünde teorik olarak tanımlanıp, benzetimler bölümünde Monte Carlo simülasyonlarının hata olasılıkları karşılaş̧ırılmıştır.

\section{Materyal ve Metot}

Sunulan analiz metodu için uygulama olarak, hipotezlere ait dağılımların bilinmediği fakat bu dağılımlarla ilişkisi olan nominal dağılımlar kümesinin, $\left\{\boldsymbol{Q}_{1}, \boldsymbol{Q}_{2}, \ldots, \boldsymbol{Q}_{\boldsymbol{M}}\right\}$, bilindiği durum seçildi. Nominal dağılımların gerçek dağılımlarla olan değişimli uzaklık ilişkisi aşağıdaki gibidir.

$$
V\left(P_{j}, Q_{j}\right) \leq \epsilon_{j}, j=1,2, \ldots, M
$$

\section{Bu denklemde $\boldsymbol{\epsilon}_{\boldsymbol{j}}$ kararll11k parametreleridir.}

Bayes çoklu hipotez test problemi doğası gereği bir hata olasılığ 1 doğurmaktadır. Bu test için ulaşılabilecek minimum üstsel hata olasılığı, bilindiği üzere ayrık hipotez dağılımları arasındaki minimum ikili Chernoff bilgisidir. İki yöntem incelenirken hata olasılıklarındaki azalma hızlarına göre incelenmiş̧ir.

Sunulan problem, genel olarak klasik kararlı hipotez testi olarak incelenmektedir. Bu problemde alfabe $\boldsymbol{X}$ sürekli veya süreksiz olabilir. Kullanılan genel yöntem minimax yaklaşımıdır ve bu yaklaşımda tüm olasılık uzayı için oluşabilecek en kötü senaryodaki hata olasılığı kullanılan uzaklığa göre minimum edilmeye çalışılmıştır [3], [5], [11], [12]. Ama bu yaklaşım çoklu 
hipotez testi için yeterli değildir ve sadece $\boldsymbol{M}=\mathbf{2}$ için kullanılabilmektedir.

\subsection{DGL Metodu}

DGL metodu, tanımlanmış problem için hata olasığına asimptotik üstel homojen dağılımlı bir sınır sunmaktadır [2]. DGL testinin temel tanımlamaları aşağıdaki gibidir.

$$
\left.A_{i, j}=\left\{x: f^{(i)}(x)>x: f^{(j)}(x)\right\}, 1 \leq i<j \leq k\right\} .
$$

Burada $A$ Borel kümesidir. $\mathcal{A}$ ise sayısı $k(k-1) / 2$ olan Borel kümeleri koleksiyonu olarak tanımlanmıştır ve bu koleksiyonun formu yukarıdaki formüldeki gibidir.

Testi tanımlamak için, deneysel ölçümler aşağıdaki gibi sunulmuştur.

$$
\mu_{n}(A)=\frac{1}{n} \sum_{i=1}^{n} \Psi_{X_{i} \in \mathcal{A}}
$$

$\Psi$, gösterge fonksiyonunu ifade etmektedir. $\mathrm{Bu}$ fonksiyon deneysel gözlem vektör dağılımını $\mathcal{A}$ setine göre her bir hipotez için karşılaştırmasını yapar. Bir sonraki aşamada ise bu karşılaştırmalara aşağıdaki test kuralı uygulanıp hipotez seçimi yapilir.

$$
\max _{A \in \mathcal{A}}\left|\int_{A} f^{(j)}-\mu_{n}(A)\right|=\min _{i=1, . ., k} \max _{A \in \mathcal{A}}\left|\int_{A} f^{(i)}-\mu_{n}(A)\right|
$$

$\mathrm{Bu}$ test yöntemi için sunduğu hata olasılığının üstel sınırı ise aşağıdaki gibidir [2].

$$
P(e) \leq 2 k(k-1)^{2} e^{-n \epsilon^{2} / 2} .
$$

DGL testi, uzunluk ölçüsü olarak $\mathrm{l}_{1}$ uzunluğu kullanmıştır. Bilindiği üzere $\mathrm{l}_{1}$ uzunluğu ile değişimli uzaklık arasında bir bağ vardır. Simülasyonlarda karşılaştırılma yapılırken $\mathrm{l}_{1}$ yerine değişimli uzaklık kullanılmıştır. $\mathrm{Bu}$ uzunluğun kullanılma nedeni ise diğer olasılıksal uzunluklarla arasındaki bağın daha geniş bir ağa sahip olmasıdır.

Literatürde çoklu hipotez testi için kullanılabilecek en uygun yöntem DGL metodudur [2]. DGL metodunda dağılımdaki belirsizlikler $Q_{j} \rightarrow P_{j}, j=1,2, \ldots, M$ şeklinde azaldığında algoritmanın hala kararlı olduğu fakat yeterli şekilde optimal duruma yaklaşamadığı Biglieri ve Gyorfi [13] tarafından gösterilmiştir. $\mathrm{Bu}$ sonuca DGL yöntemini sürekli ikilik alfabe için olabilirlik oran testi ile karşılaştırarak ulaşmışlardır. Kullandıkları olabilirlik oran testi ise aşağıdaki gibidir.

$$
T_{N} \triangleq \frac{1}{N} \sum_{n=1}^{N} \log \frac{f_{1}\left(x_{n}\right)}{f_{2}\left(x_{n}\right)}
$$

burada $\log \frac{f_{1}\left(x_{n}\right)}{f_{2}\left(x_{n}\right)}$ değerinin 0 değerinden büyük yada küçük olduğuna bakılarak karar kuralı uygulanmıştır.

\subsection{Tipler Metodu}

Temelinde tipler metodu olup Afşer tarafindan sunulan testte[15], $V\left(P_{j}, Q_{j}\right) \leq \epsilon_{j}$ koşulu altında klasik çoklu hipotez test probleminde süreksiz girdiler için genellenme olarak alınmıştır. $Q_{j} \rightarrow P_{j}$ koşulu altında testin performansı optimal test performansına yaklaşmaktadır. Afşer tarafından sunulan analizin [15] temelinde tipler yöntemi [7, Bölüm 11] bulunmaktadır. Bu yönteme dair bazı tanımlamalar ve yapılan analiz için önemli noktalar aşağıda verilmiştir.

$$
P_{x^{n}}(a) \triangleq \frac{1}{n} N\left(a \mid x^{n}\right), \quad \forall a \in X .
$$

Burada $N\left(a \mid x^{n}\right)$ terimi, $a$ sembolünün $x^{n}$ vektöründe görülme sayısını ifade eder. Tip sınıfı $T_{P}{ }^{n}$ ise tipi $P$ olan tüm $x^{n}$ vektörlerinin kümesidir.

Önsav 1: $T_{P}{ }^{n}$ kümesinin eleman sayısı, $\left|T_{P}{ }^{n}\right|$, aşağıdaki eşitsizliğe uyar.

$$
(n+1)^{-|X|} 2^{n H(P)} \leq\left|T_{P}{ }^{n}\right| \leq 2^{n H(P)} .
$$

Burada $H(P)$ terimi $P$ dağılımının entropisini gösterir.

$$
H(P)=-\sum_{x \in X} P(x) \log P(x)
$$

$P^{n} \triangleq\left\{P: T_{P}^{n} \neq \varnothing\right\}$ ise uzunluğu $n$ olan tüm tip sınıflarının kümesidir.

Önsav 2: $P^{n}$ kümesinin eleman sayısı, $\left|P^{n}\right|$, aşağıdaki eşitsizliğe uyar.

$$
\left|P^{n}\right| \leq(n+1)^{|X|}
$$

Önsav 3: Aynı tipe sahip tüm vektörler aynı olasılığa sahiptir. Eğer $x^{n}$ gözleminin elemanları $P$ dağılımından geliyorsa

$\operatorname{Pr}\left[X^{n}=x^{n}\right]=2^{-n\left(H\left(P_{x^{n}}\right)+D\left(P_{x^{n}} \| P\right)\right)}$.

Burada $D(Q \| P)$ terimi $Q$ dan $P$ 'ye Kullback Leibler (KL) uzaklığını ifade eder.

$$
D(Q \| P)=\sum_{x \in \mathrm{X}} \log \frac{Q(x)}{P(x)}
$$

\subsubsection{Klasik Tipler Metodu}

En yakın komşu kuralında, olasılık kabul bölgeleri $\Omega_{1}, \Omega, \ldots, \Omega_{M}$ ve bu bölgelerin tümleri $\Omega_{i}{ }^{c}$ olarak tanımlanır ise Sanov'un teoremi aşağıdaki hata olasılığını gösterir.

$$
\begin{aligned}
& P_{e} \leq 2^{\left(-n D\left(P_{i}^{*}, P_{i}\right)+\frac{(|X|-1) \log (n+1)}{n}\right)}, \\
& P_{i}^{*} \triangleq \operatorname{argmin}_{p \in \Omega_{i}} c D\left(p \mid P_{i}\right),
\end{aligned}
$$

Yukarıdaki sonuçları kullanarak toplam hata olasılığı aşağıdaki gibi hesaplanabilir.

$$
\begin{aligned}
& P(e)=\sum_{i=1}^{M} P\left(e \mid \mathcal{H}_{i}\right) P\left(\mathcal{H}_{i}\right), \\
& \leq \sum_{i=1}^{M} 2^{\left(-n D\left(P_{i}^{*}, P_{i}\right)-\frac{(|X|-1) \log (n+1)}{n}\right)}, \\
& \leq M \max _{i} 2^{\left(-n D\left(P_{i}^{*} \mid P_{i}\right)-\frac{(|X|-1) \log (n+1)}{n}\right)}, \\
&=2^{-n\left(\min _{i}\left(D\left(P_{i}^{*} \mid P_{i}\right)-\frac{|x-1| \log (n+1)}{n}-\frac{\log (M)}{n}\right)\right.}, \\
& \leq 2^{-n\left(\min _{i \neq j} C\left(P_{i}, P_{j}\right)-\frac{|x-1| \log (n+1)}{n}-\frac{\log (M)}{n}\right)} \\
& \quad \mathrm{i}=1,2, \ldots \mathrm{M}, \mathrm{j}=1,2, \ldots \mathrm{M},
\end{aligned}
$$

Ayrıca denklemlerin son adımında $\min _{i} D\left(P_{i}^{*} \mid P_{i}\right)$ değeri $P_{i}^{*}$ aşağıdaki formda iken $\min _{i \neq j} C\left(P_{i}, P_{j}\right)^{i}$ değerine eşit kabul edilmiştir.

$$
P_{i}^{*}=\frac{P_{i}(x)^{\lambda} P_{j}(x)^{1-\lambda}}{\sum_{x \in X} P_{i}(x)^{\lambda} P_{j}(x)^{1-\lambda}}
$$


Yukarıdaki denklemde $\lambda$ değeri $D\left(P_{i}^{*} \mid P_{i}\right)=D\left(P_{i}^{*} \mid P_{j}\right)$ olarak seçilmiştir [9].

\subsubsection{Kararlı Tipler Metodu}

Haberleşme sistemlerinde performans analizi için yapılan genel yöntem, verilen girdiler için hata olasılığının hesaplanması şeklindedir. Sistemin performansı verilen bütün girdiler için ortalama hata olasılığ hesaplanarak bulunur. Burada verilmiş $\vec{x}$ olasıllı tipi için, $P_{\vec{x}}$, durumu incelenmiştir. Bu bağlamda $\vec{x} \in$ $T\left(P_{\vec{x}}\right)$ için $P\left(e \mid P_{\vec{x}}\right)$ ortalama hata olasılığı olarak tanımlandı.

$$
P\left(e \mid P_{\vec{x}}\right) \triangleq \sum_{\vec{x} \in \mathrm{T}\left(P_{\vec{x}}\right)} P(e \mid P(\vec{x})) P(\vec{x}),
$$

Ortalama hata olasılıkları $\vec{x} \in \mathcal{X}^{n}$ üzerinden hesaplanabilir.

$$
\begin{aligned}
P(e) & =\sum_{\vec{x} \in X^{n}} P(e \mid P(\vec{x})) P(\vec{x}), \\
& =\sum_{T\left(P_{\vec{x}}\right) \in \mathcal{P}^{n}} \sum_{\vec{x} \in T\left(P_{\vec{x}}\right)} P(e \mid P(\vec{x})) P(\vec{x}), \\
& =\sum_{T\left(P_{\vec{x}}\right) \in \mathcal{P}^{n}} P(e \mid P(\vec{x})) .
\end{aligned}
$$

Eğer $P(e \mid P(\vec{x})$ teriminin $P(\vec{x})$ 'e bağlı belirli azalan üssel parçaları varsa, aralarından minimum olanı $P(e)$ değerini domine eder. İlerleyen savlarda, bu durumu içeren olayı kanitliyor.

Önsav 4:

$$
P\left(e \mid P(\vec{x}) \leq 2^{-n\left(\min _{i \neq j} \max \left\{D\left(P(\vec{x}) \| P_{i}\right), D\left(P(\vec{x})|| P_{j}\right)\right\}-\frac{\log M}{n}\right)} .\right.
$$

Önsav 5:

$\min _{T\left(P_{\vec{x}}\right) \in \mathcal{P} n} \max \left\{D\left(P(\vec{x}) \| P_{i}\right), D\left(P(\vec{x}) \| P_{j}\right)\right\}=C\left(P_{i}, P_{j}\right)$.

$P_{\vec{x}}$ aşağıdaki formda minimize edilmiştir.

$$
P_{\vec{x}}^{\lambda}=\frac{P_{i}(x)^{\lambda} P_{j}(x)^{1-\lambda}}{\sum_{x \in \mathcal{X}} P_{i}(x)^{\lambda} P_{j}(x)^{1-\lambda}}
$$

$\lambda$ değeri $D\left(P_{\vec{x}}{ }^{\lambda} \mid P_{i}\right)=D\left(P_{\vec{x}}{ }^{\lambda} \mid P_{j}\right)$ şeçildi.

$P\left(e \mid P_{\vec{x}}\right)$ ifadesinin, $\min _{i \neq j} \max \left\{D\left(P(\vec{x}) \| P_{i}\right), D\left(P(\vec{x}) \| P_{j}\right)\right\}$ üstsel ifadesine bağlı olarak azalmakta olduğu Önsav 4 'te gösterildi. Önsav 5 ise belirlenmiş tipler üstündeki bu terimin ulaşabileceği en küçük değerin $\min _{i \neq j} C\left(P_{i}, P_{j}\right)$ olduğu sunuldu.

Sonuç olarak, $x \in \mathcal{X}$ için ortalama hata olasıllı̆ına üst sınır aşağıdaki gibi konuldu.

$$
\begin{aligned}
& P(e) \leq\left|\mathcal{P}^{n}\right| \max _{T\left(P_{\vec{x}} \in \mathcal{P}^{n}\right)} P\left(e \mid P_{\vec{x}}\right), \\
& \leq 2^{-n\left(\min _{i \neq j} \min _{T\left(P_{\vec{x}}\right) \in \mathcal{P} n} \max \left\{D\left(P(\vec{x}) \| P_{i}\right), D\left(P(\vec{x}) \| P_{j}\right)\right\}-\frac{\log M}{n}\right)}, \\
& \leq 2^{-n\left(\min _{i \neq j} C\left(P_{i}, P_{j}\right)-\frac{|X-1| \log (n+1)}{n}-\frac{\log M}{n}\right)}, \\
& i=1,2, \ldots, M ; j=1,2, \ldots, M .
\end{aligned}
$$

Tipler metoduna dayalı analiz, gerçek dağılımları edinmek amacıyla nominal dağılımların üstünde yapılan yuvarlama operasyonuna dayanmaktadır. Gerçek dağılımlar yerine onları simgeleyen $\overline{P_{1}}, \overline{P_{2}}, \ldots, \overline{P_{M}}$ terimleri, $Q_{1}, Q_{2}, \ldots, Q_{M}$ terimlerinden aşağıdaki gibi türetildi.

$$
\bar{P}_{j}(x)=\frac{Q_{j}(x)+\epsilon_{j}}{1+|X| \epsilon_{j}} \quad \forall x \in X .
$$

$\bar{P}_{j}$ 'nin uygun bir dağılım olduğu, $\mathcal{X}$ alfabesi üstüne yapılan toplama işlemiyle görülür.

$$
\sum_{x \in \mathcal{X}} \bar{P}_{j}(x)=\frac{\sum_{x \in X}\left(Q_{j}(x)+\epsilon_{1}\right)}{1+|X|}=\frac{1+|X| \epsilon_{1}}{1+|X| \epsilon_{1}}=1
$$

Eğer $P_{j}(x) \leq 1$ ise $\forall x \in \mathcal{X}$ için yukarıdaki denklem sağlanır. $\mathcal{X}$ alfabesi için eş oranlı bir dağılıma sahip $U$ dağılımı aşağıdaki gibi tanımlanırsa,

$$
U(x) \triangleq \begin{cases}\frac{1}{|\mathcal{X}|} & x \in \mathcal{X}, \\ 0 & x \notin \mathcal{X},\end{cases}
$$

Bu tanımlama ışı̆̆ında, $\bar{P}_{j}(x)$ 'in $Q_{j}(x)$ etrafinda yuvarlanmış versiyonu olduğu ve $\epsilon_{j}$ arttıkça $U$ dağılımına genişlediği görülebilir. Bunu göstermek için, $\bar{P}_{J}(x)>Q_{j}(x)$ koşulunun sağlandığı aşağıdaki denklemlerde gösterildi.

$$
\begin{aligned}
\frac{Q_{j}(x)+\epsilon_{j}}{1+|X| \epsilon_{j}} & >Q_{j}(x), \\
Q_{j}(x)+\epsilon_{j} & >Q_{j}(x)+Q_{j}(x)|X| \epsilon_{j}, \\
\frac{1}{|X|} & >Q_{j}(x) .
\end{aligned}
$$

Böylece, $\quad Q_{j}(x)<\frac{1}{|x|} \quad$ koşulunda, $\quad \bar{P}_{j}(x)>Q_{j}(x)$ eşitsizliğinin sağlandığı gözlemlendi. Benzer şekilde $Q_{j}(x)>$ $\frac{1}{|x|}$ koşulunda $\bar{P}_{J}(x)<Q_{j}(x)$ sağlanıyor. Sonuç olarak $\epsilon_{j}$ değeri arttığ sürece, $\bar{P}_{j}(x)$ değerinin $Q_{j}(x)$ değerinden uzaklaşı $U$ değerine yaklaşmakta olduğu ve $\epsilon_{j}$ değeri azaldığı durumda ise $Q_{j}(x)$ değerine yaklaştığ gözlemlendi.

$$
\lim _{\epsilon_{j} \rightarrow \infty} \overline{\bar{P}}_{J}(x)=\lim _{\epsilon_{j} \rightarrow \infty} \frac{Q_{j}(x)+\epsilon_{j}}{1+|\mathcal{X}| \epsilon_{j}}=U(x) .
$$

Öte yandan, $\epsilon_{j}$ değeri düşerken $\bar{P}_{j}(x)$ değeri $P_{j}(x)$ değerine yaklaşmaktadır.

$$
\lim _{\epsilon_{j} \rightarrow 0} \bar{P}_{j}(x)=\lim _{\epsilon_{j} \rightarrow 0} \frac{Q_{j}(x)+\epsilon_{j}}{1+|X| \epsilon_{j}}=Q_{j}(x)=P_{j}(x) .
$$

$\bar{P}_{j}(x)$ dağılımının kullanılmasındaki ana neden; $P_{j}$ üzerinden üretilen $\vec{x}$ için $P(\vec{x})$ değerine üst sınır sağlamasından kaynaklanmaktadır. Bu durumu Önsav 6'da gösterildi.

Önsav 6: $\forall \vec{x} \in T\left(P_{\vec{x}}\right), V\left(P_{j}, Q_{j}\right) \leq \epsilon_{j}$ olduğu biliniyorsa ve $P_{j}$ 'den bağımsız ise,

$$
P_{j}(x) \leq 2^{-n\left(H P(\vec{x})+\mathrm{D}\left(P(\vec{x}) \| \mid \overline{P_{j}}\right)-\log \left(1+|X| \epsilon_{j}\right)\right)} .
$$

$\vec{x}$ 'nün tam olarak bilinmediği $P_{j}$ gerçek dağılımlarından üretilmiş dağllım altında Önsav 6, Önsav 3 'ün genellenmiş halidir. Fakat bu genelleme $V\left(P_{j}, Q_{j}\right) \leq \epsilon_{j}$ koşulunun bilindiği durum için geçerlidir. Ayrıca $\epsilon_{j} \rightarrow 0$ durumunda Önsav 6'nın Önsav 3 ile eşleştiğinden önemsenmelidir. Çünkü en kötü durumdaki hata üstünün hipotez çiftleri arasındaki Chernoff bilgisine eşit olduğunu göstermektedir.

Afşer'in sunduğu test ve hata olasıllğına getirilen üst sınır aşağıdaki teorem de gösterildi. 


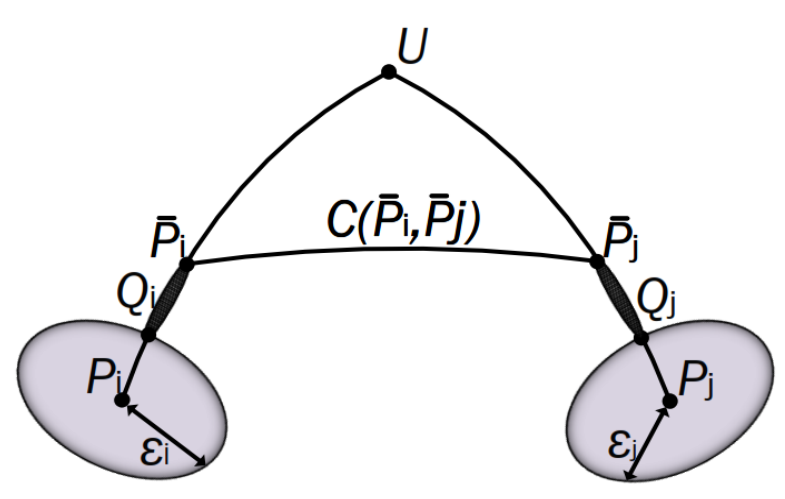

Şekil 1. Tipler metoduna dayalı analizin geometrik gösterimi.

Teorem 1: Bayes çoklu hipotez test problemi için, karar kuralının toplam hata olasılığı

$$
\operatorname{Seç} \mathcal{H}_{j}, j=\operatorname{argmin}_{i \in\{1,2, \ldots, M\}} D\left(P_{\vec{x}} \mid \bar{P}_{l}\right) .
$$

Üst sınırı ise,

$$
\begin{gathered}
P(e) \leq 2^{-n\left(\left(\min _{i \neq j}\left(P_{i}, P_{j}\right)-\log (1-|X| \epsilon)\right)-\frac{|X-1| \log (n+1)}{n}-\frac{\log M}{n}\right)} \\
i=1,2, \ldots, M ; j=1,2, \ldots, M .
\end{gathered}
$$

şeklindedir. Denklemdeki seçilen $\epsilon$ ise aşağıdaki kurala göre seçilmektedir,

$$
\epsilon \triangleq \max _{k} \epsilon_{k}, \quad k=1,2, \ldots, M .
$$

Bu problemin geometrik açıklaması Şekil 1'de gösterilmiştir. $V\left(P_{j}, Q_{j}\right) \leq \epsilon_{j}$ kısıtlaması $Q_{j}$ nominal dağılımı için belirsizlik bölgesi yaratıyor. $\mathrm{Bu}$ bölgenin merkezinde $P_{j}$ olduğu varsayılıyor. Bu yapı $\epsilon_{j}$ küçüldükçe, $Q_{j}$ dağılımını $P_{j}$ dağılımına yaklaştırıyor. Benzer şekilde $\epsilon_{j}$ küçüldükçe $\bar{P}_{j}$ değerini ise $Q_{j}$ değerine yaklaştırıyor. Denklem 3 'teki $\min _{i \neq j} C\left(P_{i}, P_{j}\right)-\log (1-$ $|X| \epsilon)$ terimde $\epsilon \ll 0$ için $\log (1-|X| \epsilon) \rightarrow 0$ olur. Böylece tipler metoduna dayalı analiz için üst sınır yaklaşık olarak hipotezler arasındaki Chernoff bilgisine eşit oluyor.

$$
\begin{aligned}
\min _{i \neq \mathrm{j}} C\left(P_{i}, P_{j}\right) & \geq \min _{i \neq \mathrm{j}}-\frac{1}{2} \ln \left(1-V\left(Q_{i}, Q_{j}\right)^{2}\right) \\
& \geq \min _{i \neq \mathrm{j}} \frac{1}{2} V\left(Q_{i}, Q_{j}\right)^{2}
\end{aligned}
$$

Üstteki eşitliklerde sol taraf dağılımlar için minimum ikili Chernoff bilgisini ifade ediyor. Denklem 4'ün sağ tarafı ise DGL testinde [2] dağılımlarda belirsizliğin olmadığı durum için, $P_{j}=$ $Q_{j}, j=1,2, \ldots, M$, hata üst sınırını ifade ediyor. $\ln (z) \leq z-$ $1, z \geq 0$ eşitsizliğine göre düzenlenerek elde edilen Denklem 5 ise bize tipler metoduna dayalı analizin $\epsilon \ll 0$ durumda hata üstel sınırının, DGL metoduna göre daha fazla olduğunu göstermektedir.

Tablo 1. Simülasyonda kullanılan degişkenler ve olasılık daılımları

\begin{tabular}{|c|c|c|c|c|}
\hline Simülasyon & $\epsilon=0.10$ & $\epsilon=0.03$ & $\epsilon=0.003$ & $\epsilon=0.0003$ \\
\hline$Q_{1}$ & {$[0.04,0.76,0.2]$} & {$[0.11,0.82,0.07]$} & {$[0.102,0.801,0.097]$} & {$[0.1003,0.7997,0.1]$} \\
\hline$Q_{2}$ & {$[0.24,0.3,0.46]$} & {$[0.29,0.23,0.48]$} & {$[0.303,0.198,0.499]$} & {$[0.3001,0.2002,0.4997]$} \\
\hline$Q_{3}$ & {$[0.7,0.05,0.25]$} & {$[0.63,0.09,0.28]$} & {$[0.599,0.098,0.303]$} & {$[0.600,0.1003,0.2997]$} \\
\hline$Q_{4}$ & {$[0.37,0.5,0.13]$} & {$[0.38,0.43,0.19]$} & {$[0.398,0.403,0.199]$} & {$[0.3999,0.3998,0.2003]$} \\
\hline$Q_{5}$ & {$[0.34,0.5,0.16]$} & {$[0.32,0.57,0.11]$} & {$[0.301,0.602,0.097]$} & {$[0.3003,0.5998,0.0999]$} \\
\hline $\min _{i \neq j} C\left(P_{i}, P_{j}\right)$ & 0.0016 & 0.0169 & 0.0327 & $8.996 e-04$ \\
\hline $\log (1+|X| \in)$ & 0.2624 & 0.0862 & 0.0090 & 0.0050 \\
\hline
\end{tabular}

\section{Araştırma Sonuçları ve Tartışma}

\subsection{Benzetimler}

$\mathrm{Bu}$ makalede gerçek olasılık dağılımları bilinmediği durum için, gösterilmiş iki metodun Monte Carlo simülasyon performansları optimal durumdaki performanslarıyla karşılaştırılmıştır. $\mathrm{Bu}$ benzetimlerde $n$ değeri arttırılarak $\log \left(P_{e}\right)$ 'nin azalışı gözlemlendi. Ayrıca tipler metoduna dayalı analizin performansı $P_{i}$ dağılımlarının bilindiği ideal test performansıyla, denklem 1, Şekil 2'de kıyaslandı. Yapılan simülasyonlarda karşılaştırılan hipotez sayısı beş, $M=5$, ve alfabedeki elaman sayısı üç olarak alınmıştır, $|X|=3$. Hipotezlere ait olasılık dağılımları aşağıdaki gibi alınmıştır.

$$
\begin{gathered}
P_{1}=[0.1,0.8,0.1], P_{2}=[0.3,0.2,0.5], P_{3}=[0.6,0.1,0.3] \\
P_{-} 4=[0.4,0.4,0.2], \quad P_{-} 5=[0.3,0.6,0.1]
\end{gathered}
$$

$\mathrm{Bu}$ dağılımlardan rastgele olarak üretilmiş deneysel dağılımlar, $Q$, ve simülasyon parametreleri Tablo 1'de gösterilmiştir. Sunumu basitleştirmek için $\epsilon_{1}=\epsilon_{2}=\cdots=\epsilon_{5}=$ $\epsilon$ alınmıştır. Ayrıca sunulan metotların serbestlik derecesi beştir. Simülasyonlarda, $\vec{x}$ rastgele şekilde $P_{i}$ dağılımlarına göre oluşturulurdu ve hipotezlerin her birinin seçilme olasılıkları eşit sekilde $\mathcal{H}_{i}=0.2, i=1,2, \ldots, 5$ alınd. $\quad P\left(e \mid \mathcal{H}_{i}\right), i=1,2, \ldots, 5$ e-ISSN: 2148-2683 değerleri her hipotez için 100 hata gözlemlene kadar devam ettirildi ve hesapland.

DGL metodu ve tipler metoduna dayalı analizin, simüle edilmiş hata olasılıklarının $n$ 'in arttırılması ile olan ilişkisi Şekil 3 'te gösterilmiştir. $\mathrm{Bu}$ şekilde, bulunan değerlerin negatif eğrisinin $\epsilon$ değeri azaldıkça optimum duruma yaklaştığ görülmektedir. Aynı zamanda tipler metoduna dayalı metodun, DGL metoduna göre $\epsilon<0.03$ için üstel azalma hızında daha iyi performans sunduğu gözlemlendi.

Afşer'in önerdiği metodun hata olasılıkları Şekil 2'de üst sinırlarıyla beraber sunulmuştur. Buradaki negatif eğri toplam hata olasılığı ve üst sınırlar $\epsilon=0.0005$ için gösterilmektedir. Aynı zamanda, sunulmuş metodun teorik sinırlarla örtüştüğü kanıtlanmıştır. Sunulan düzenekte $\min _{i \neq j} C\left(P_{i}, P_{j}\right)=0.0329$ 'dur ve sunulan testin $\epsilon<0.03$ için kabul edilebilir bir performans sergilediği gösterilmiştir. 


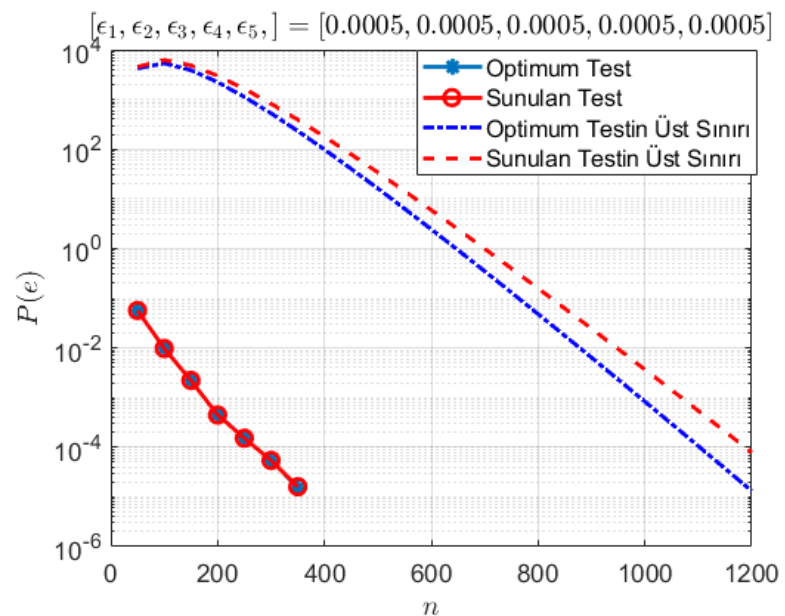

Şekil 2. $\in=0.0005$ için, optimum ve deneysel hata olasılıklarının n ile olan ilişkisi.

\section{Sonuç}

DGL metodunun hizının $n \rightarrow$ inf durumunda tipler metoduna dayalı analiz yöntemine göre hızının yavaşladığı gösterildi. Tipler metoduna dayalı analizin, gerçek hipotez dağılımlarının bilinmediği fakat nominal dağılımlarla ilişkisinin değişimli uzaklığı, $\epsilon$, bilindiği durumda çoklu Bayes hipotez problemi için bir genelleme olduğu yapılan simülasyonlarla deneysel olarakta gösterildi. Ek olarak, Afşer tarafından sunulan yöntemdeki yuvarlamanın operasyonu $\epsilon$ azaldıkça, $\overline{P_{M}} \rightarrow Q_{M} \rightarrow$ $P_{M}$ durumunu sağladığ

Tipler metoduna dayanan analizin performansının, DGL metodunu geçtiği aralık tam olarak $0.015<\epsilon<0.02$ 'dir. Buradaki epsilon değeri yapılan simülasyonlarla bulundu. Nominal dağılımlar ve gerçek dağılımlar arasındaki uzunluk değişimli uzaklık olarak varsayıldı. Çünkü değişimli uzaklık $\mathrm{l}_{1}$, Hellinger, Wassertein, $X^{2}$ ve KL uzaklıkları ile sınırlanabiliyor [14]. Bu sınırlandırmalar testin uygulanabilirliğini arttırmaktadır.

\section{Kaynakça}

[1] E. L. Lehmann and J. P. Romano, Testing statistical hypothesis (Springer Texts in Statistics), Springer-Verlag, 2005.

[2] L. Devroye, L. Gyorfi and G. A. Lugosi, A note on robust hypothesis testing, IEEE Transactions on Information Theory, 48 (7), 2111-2014, 2002.

[3] P. J. Huber, Peter J. "A Robust Version of the Probability Ratio Test", Ann. Math. Statist. 36 (1965), no. 6, 1753-1758.

[4] B. C. Levy, 'Robust Hypothesis Testing With a Relative Entropy Tolerance," IEEE Trans. Inf. Theory, vol. 55, no. 1, pp. 413-421, Jan. 2009.

[5] C. Levy, Principles of signal detection and parameter estimation, Springer Publishing Company, 2008.

[6] G. Gül and A. M. Zoubir,'Minimax Robust Hypothesis Testing," in IEEE Transactions on Information Theory, vol. 63, no. 9, pp. 5572-5587, Sept. 2017, doi: 10.1109/TIT.2017.2693198.

[7] T. Cover M. and J. Thomas A., Elements of Information Theory, 2nd ed. John Wiley I\& Sons, 2012.

[8] C. C. Leang and D.H.Johnson On the asymptotic of Mhypothesis Bayesian detection, IEEE Transactions on Information Theory, 43 (1): 280-282, 1997.

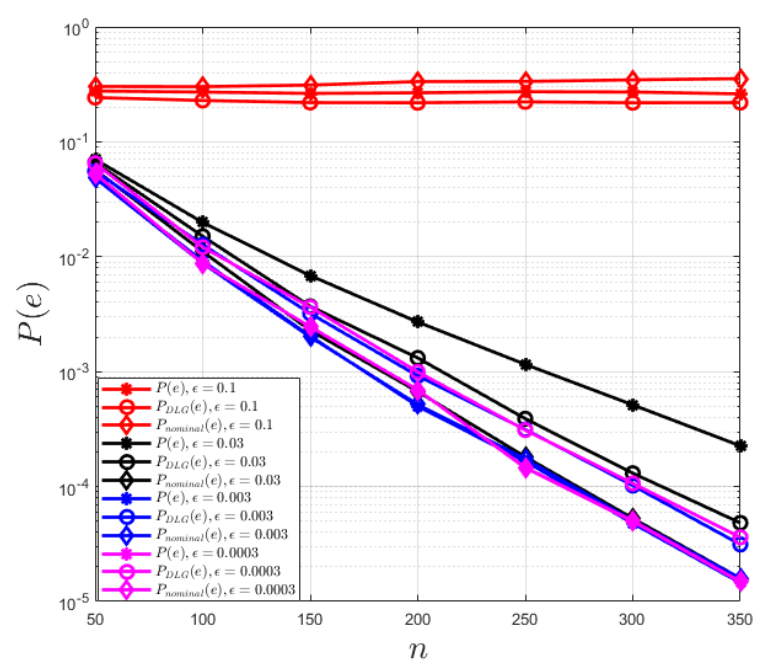

Şekil 3. Hata olasılıklarının n ile olan ilişkisi.

Denklem 2'de bulunan $\min _{i \neq j} C\left(P_{i}, P_{j}\right)-\log (1+|X| \epsilon)$ teriminin negatif olması durumunda, sunulan testin ulaşılabilir bir üst sınır sunamamasına rağmen testin kabul edilebilir bir performans sunduğu Tablo 1'de ve Şekil 3'de gösterilmiştir.

Şekil 3'te hata olasılıkları görülmektedir. Burada $n$ arttırılarak, tipler metoduna dayalı analizin yuvarlama opearasyonu yapılmış logaritmik hata olasılığını $P(e)$, yuvarlama operasyonu yapılmamış logaritmik hata olasılığını $P_{\text {nominal }}(e)$ ve DGL metodunun logaritmik hata olasılıkları $P_{D G L}(e)$ görülmektedir. $\epsilon$ değeri azaldıkça tipler metoduna dayalı yöntemin daha iyi çalıştığı görülmüştür. Ayrıca yuvarlanma operasyonu yapılmış dağılımların performansının, yapılmamış nominal dağılım performansını $\epsilon<0.0003$ durumunda geçtiği gözlemlenmiştir.

[9] M. B. Westover, Asymptotic geometry of multiple hypothesis Testing, IEEE Transactions on Information Theory, 54 (7), 3327-3329, 2008.

[10]Chernoff, Herman. “A Measure of Asymptotic Efficiency for Tests of a Hypothesis Based on the sum of Observations", Ann. Math. Statist. 23 (1952), no. 4, 493-507.

[11]P. J. Huber and V. Strassen, "Robust confidence limits," Z. Wahrchein- lichkeitstheorie verw. Gebiete, vol. 10, pp. 269278, 1968.

[12]P. J. Huber, Robust Statistics, New York J. Wiley, 1981.

[13]E. Biglieri and L. Gyorfi, Some remarks on robust binary hypothesis testing, IEEE International Symposium on Information Theory, 566-570, 2014.

[14]A. L. Gibbs and F. E. Su, On Choosing and bounding the probability metrics, International Statistical Review, 70 (3), 419-435, 2002.

[15]H. Afşer, Some Remarks on Bayesian Multiple Hypothesis Testing, Hacettepe Journal of Mathematics \& Statistics, under revision. 